Cómo citar este artículo en Chicago: Dalmau, Iván Gabriel. "Michel Foucault y el problema del método: reflexiones en torno a la arqueo-genealogía”. Escritos 29, no. 62 (2021): 84-100. doi: http://dx.doi.org/10.18566/escr.v29n62.a06

\title{
Michel Foucault y el problema del método: reflexiones en torno a la arqueo-genealogía
}

\author{
Michel Foucault and the Issue of Method: Perspectives on Archaeo-genealogy
}

Iván Gabriel Dalmau' ${ }^{1}$ (])

\begin{abstract}
RESUMEN
El propósito de este trabajo es revisar la problematización foucaultiana del método en su concepción de la filosofía como actividad de diagnóstico crítico del presente. En primer lugar, reconstruiremos la manera en que el filósofo problematiza la crítica como actividad de diagnóstico y se enfoca de modo recurrente en el trabajo de archivo sobre el discurso de las ciencias humanas. Luego, nos detendremos en la problematización arqueológica del método y en lo que denominamos "ecos arqueológicos de la genealogía". Por tanto, mostraremos la manera en que la problematización de las ciencias humanas en tanto saberes habilita una forma de crítica articulada por la preocupación de diagnosticar el presente. Por esta razón, nos enfocaremos en el desplazamiento introducido por la perspectiva foucaultiana desde la pregunta normativa acerca de la objetividad cognoscitiva de las ciencias humanas hacia una crítica ontológico-política. Modo de crítica que, preocupada por diagnosticar el presente, caracterizamos como una arqueogenealogía de las formas de objetivación.
\end{abstract}

Palabras clave: Michel Foucault; Arqueología; Genealogía; Método; Crítica; Saber; Ciencias humanas; Historia; Filosofía; Diagnóstico.

\begin{abstract}
The purpose of this paper is to review Foucault's problematization of method within the framework of his conception of philosophy as a critical diagnostic activity of the present. First, we will reconstruct the way in which the philosopher problematizes the critique as a diagnostic activity and focuses repeatedly on archival work on the discourse of the human sciences. Then, we will focus on the archaeological problematization of method and what we call "archaeological echoes of genealogy". Therefore, we will show the way in which the problematization of the human sciences as knowledge enables a form of criticism articulated by the concern to diagnose the present. For this reason, we will focus on the displacement introduced by Foucault's perspective from the normative question about the cognitive objectivity of the human sciences towards an ontological-political critique. Mode of criticism that, concerned with diagnosing the present, we characterize as an archeo-genealogy of the forms of objectification.
\end{abstract}

Keywords: Michel Foucault; Archaeology; Genealogy; Method; Knowledge; Human Sciences; History; Philosophy; Diagnosis.

1 Doctor en Ciencias Sociales por la Universidad de Buenos Aires, Argentina. Profesor adjunto de Epistemología de las Ciencias Sociales en la Escuela de Humanidades de la Universidad Nacional de San Martín, Argentina. Correo electrónico: ivandalmau@yahoo.com.ar. 


\section{Consideraciones preliminares}

En retrospectiva, los trabajos publicados aparecen como fotografías, como recortes momentáneos de un proceso [...]. Sin embargo, la lectura de las lecciones del Collège de France provee un antídoto eficiente para esto. En dichas conferencias vemos a Foucault trabajando, volviendo constantemente sobre cuestiones previas, retomándolas y reformulándolas. ${ }^{2}$

Este artículo se inscribe en el dosier temático centrado en las formas de problematización del método de trabajo filosófico que tuvieron lugar en el seno de las distintas vertientes de la filosofía contemporánea, desde la filosofía anglosajona de cuño analítico, hasta las distintas modulaciones de la filosofía francesa posnietzscheana, pasando por las reflexiones desarrolladas en la tradición fenomenológica y hermenéutica. ${ }^{3}$ Específicamente, nos detendremos en la indagación de las "cuestiones de método" desplegadas en los trabajos del filósofo francés Michel Foucault. En ese sentido, en consonancia con la cita que hemos puesto como epígrafe, reconstruiremos el modo en que el pensador francés problematiza de manera recurrente la forma en que metodológicamente despliega su trabajo de archivo. Es decir que, apoyados en el característico gesto foucaultiano de reelaboración y reformulación recurrente de sus trabajos precedentes, ${ }^{4}$ nos detendremos en sus reflexiones recurrentes respecto del método de trabajo filosófico desplegado en las canteras de la historia y jalonado por preocupaciones ontológico-políticas vinculadas a una concepción de la crítica como actividad de diagnóstico del presente.

Cabe destacar que el ejercicio de lectoescritura que desplegaremos a continuación se encuentra posibilitado por la transformación del material disponible producida, fundamentalmente, como fruto de la publicación en formato libro de los cursos dictados por Foucault en el Collège de France. ${ }^{5}$ La edición de los cursos permitió completar los años de "silencio editorial", que, en lo que a la publicación de libros en vida por parte del autor respecta, se extendía entre la publicación de Larchéologie du savoir en 1969 y Surveiller et punir en 1975, y entre el primero y el segundo y tercer tomo de Histoire de la sexualité. De este modo, para decirlo foucaultianamente, la transformación del corpus formado por el conjunto de los documentos publicados en nombre de Michel Foucault cambió el modo en que este aparecía (de manera inmanente al tejido documentario) como un autor susceptible de ser estudiado en el ámbito académico de la historia de la filosofía contemporánea. Modificación en el corpus documental que nos conduce a tomar distancia de la manera en que se constituyó tempranamente en la bibliografía secundaria la caracterización de Foucault como un "autor" en cuya "obra" podrían distinguirse tres etapas, ${ }^{6}$ vinculadas

2 Sven-Olov Wallenstein, "Introduction: Foucault, Biopolitics, and Governmentality", en Foucault, Biopolitics and Governmentality, ed. por Sven-Olov Wallenstein y Jakob Nilsson (Huddinge: Södertörn University, 2013), 10.

3 En primer lugar, quisiera agradecer a Francisco Díez Fischer y a Claudio Cormick por brindarme la posibilidad de participar de este dosier. Por otra parte, no puedo dejar de expresar mi agradecimiento para con mi director (como tesista de grado en Filosofía y como posdoctorando en Ciencias Sociales), Marcelo Raffin.

4 Alessandro Fontana y Mauro Bertani, "Situation du Cours", en "Il faut défendre la société": Cours au Collège de France, 1976, Michel Foucault. París: Éditions Gallimard, 1997), 248.

5 Vanessa Lehm y Miguel Vatter, "Introduction”, en The Government of Life: Foucault, Biopolitics and Neoliberalism, ed. por Vanesa Lehm y Miguel Vatter (Nueva York: Fordham University Press, 2014), 1-2; Marcelo Raffin, "La verdad y las formas políticas: La lectura temprana de la tragedia de Edipo en Michel Foucault", Anacronismo e Irrupción 5, n. ${ }^{\circ}$ 8 (2015): 54-78.

$6 \mathrm{Al}$ respecto, se destacan, entre otros, trabajos desarrollados en la década de 1980 por reconocidas figuras del campo intelectual francés y estadounidense. Hubert Dreyfus y Paul Rabinow, Michel Foucault: Beyond Structuralism and 
a décadas, métodos y objetos. Específicamente, desde dicho marco de lectura, se consideraba que en la década de 1960 Foucault se valía del método arqueológico y su objeto privilegiado de estudio era el saber, de ahí que la presunta etapa sea denominada "arqueológica". Tras lo cual, durante la década de 1970 se habría ocupado de problematizar el poder, valiéndose de la genealogía nietzscheana como método. Por último, en la década de 1980, el filósofo redirigiría su preocupación hacia la constitución ética de la subjetividad, periodo en el que supuestamente modificaría el recorte temporal de su archivo, al enfocarse en discursos formados en el seno de la filosofía y la cultura grecorromana.

Desde un punto de vista formal, dividiremos el artículo en tres apartados. En el primero, nos ocuparemos de revisar el modo en que el filósofo problematiza la filosofía como actividad de diagnóstico crítico del presente y la manera recurrente en que el discurso de las ciencias humanas se erige como blanco de su trabajo de archivo. Tras lo cual, en el segundo apartado, dirigiremos el foco de lectura hacia la problematización de las "cuestiones de método" sobre las que el pensador asienta su producción filosófico-crítica desplegada en las canteras de la historia. Finalmente, cerraremos el artículo con un breve apartado de reflexión.

\title{
Michel Foucault: la filosofía como actividad de diagnóstico
}

\begin{abstract}
Hacer la historia de la objetivación, no explicar lo que se ha pensado acerca de la locura en la modernidad, no cómo las sociedades occidentales se han comportado con respecto a ella, sino cómo se ha constituido un objeto de saber y de práctica social y política que tenía los caracteres de la locura; por lo tanto, hacer la historia de la constitución de los objetos significaba, para Foucault, contribuir a la problematización del presente. ${ }^{7}$
\end{abstract}

A modo de antesala de la reconstrucción de las reflexiones acerca de las "cuestiones de método" elaboradas por el filósofo a lo largo de su producción, tal como lo explicitáramos en la introducción, en este apartado, nos proponemos revisar la forma en que Foucault problematiza la filosofía como actividad de diagnóstico y el abordaje recurrente de los saberes acerca de "lo humano" que el filósofo realiza como blanco del trabajo de archivo jalonado por la preocupación respecto de la constitución ontológicopolítica de la actualidad. ${ }^{8}$ Sin embargo, antes de proseguir con la lectura, consideramos pertinente realizar la siguiente aclaración metodológica, a saber: apoyarnos en la manera en que Foucault alude a otros filósofos y corrientes filosóficas desde ningún punto de vista implica comprometernos con la plausibilidad filológica e histórico-conceptual de la interpretación foucaultiana. Es decir que, si bien consideramos relevante el modo en que, por ejemplo, los nombres de Kant y Nietzsche se encuentran presentes en el discurso foucaultiano, articulando su economía, no implica en absoluto convalidar en

Hermeneutics, 2. ${ }^{\mathrm{a}}$ ed. (Chicago: University of Chicago Press, 1983); Maurice Blanchot, Michel Foucault tel que je l’imagine (París: Éditions Fata Morgana, 1986); Gilles Deleuze, Foucault. París: Les Éditions de Minuit, 1986.

7 Claudia Giordano, "Un’idea di filosofia: Michel Foucault, dalla critica dell'antropologia all'etica de la cura di sé” (tesis de doctorado, Scuola di Alta Formazione nelle Scienze Umane e Sociali dell’Università degli Studi di Napoli Federico II, 2007): 70.

8 Giovanni Maria Mascaretti, "Michel Foucault on Problematization, Parrhesia and Critique", Materiali Foucaultiani 3 , n. ${ }^{\circ}$ 5-6 (2014): 139. 
un registro historiográfico lo atinada o plausible que resulta la manera en que el francés se ubica frente a ambos filósofos alemanes. En otros términos, son dos problemas de investigación diferentes: cómo entiende la filosofía crítica Foucault (cuestión que requiere revisar el modo en que se ubica en torno al legado de Kant y Nietzsche) y cómo lee Foucault a Kant y a Nietzsche. Remarcamos que no puede pasarse, sin solución de continuidad, de un problema al otro.

Retornando al interrogante que articula la escritura de este apartado, tomamos, entonces, como punto de partida, la lectura propuesta por el pensador en la primera clase del curso dictado en el Collège de France durante el ciclo lectivo 1982-1983, respecto del modo en que Kant respondió a la pregunta "Was ist Aufklärung?", para inscribir su propia labor en una modulación de la crítica:

Me parece que la elección filosófica a la que nos encontramos confrontados actualmente es esta. Hay que optar por una filosofía crítica que se presentará como una filosofía analítica de la verdad en general, o por un pensamiento crítico que tomará la forma de una ontología de nosotros mismos, de una ontología de la actualidad. ${ }^{9}$

En dicha clase, frente a la realización de una "analítica de la verdad en general" (preocupada por las posibilidades del conocimiento y sus límites infranqueables), Foucault reivindica la práctica de la crítica como una ontología de la actualidad. Para clarificar su concepción de la crítica, propone una breve reconstrucción de la filosofía posterior a Kant, distinguiendo dos posteridades críticas. Por un lado, tendríamos un variopinto conjunto de corrientes filosóficas, que, haciéndose eco del Kant de la Crítica de la razón pura, habrían desplegado una forma de trabajo filosófico en que la crítica tendría como aspecto clave detectar los límites de las posibilidades del conocimiento, de modo tal de fundamentar qué puede conocerse y cómo, y así forjar un triángulo en que las nociones de crítica, fundamentación y normación se llamarían la una a la otra. En contraposición, Foucault reivindicará la inscripción de sus trabajos en la otra posteridad crítica erigida en torno al legado kantiano, referido particularmente al Kant que, en tanto filósofo, realiza una interrogación crítica respecto de su propio presente en el clásico opúsculo sobre la Aufklärung. ${ }^{10}$ Posteridad de la crítica, que, motorizada por la preocupación respecto de la constitución del presente de quien filosofa, atravesaría los trabajos de Hegel de la escuela de Fráncfort a Nietzsche y Weber.

Si bien es conocido que Foucault se ha ocupado en reiteradas oportunidades (entre 1978 y 1984) de revisar la lectura kantiana de la Aufklärung, la reconstrucción de los matices y las declinaciones de tales lecturas quedan por fuera de los objetivos de nuestro trabajo. Por el contrario, dado que el objetivo de este apartado es reponer el modo en que caracteriza el ejercicio de la crítica como actividad de diagnóstico, como actitud filosófica que enmarca su abordaje recurrente del discurso de las ciencias humanas, en pos de poner nuestra reconstrucción al abrigo de la eventual objeción de que esta caería en una suerte de forzamiento teleológico, consideramos pertinente revisar referencias previas de Foucault a la práctica de la filosofía como actividad de diagnóstico. En ese sentido, cabe destacar que en 1966, en el contexto de la publicación de Les mots et les choses, brindará una entrevista en la que, si bien armará un juego de oposiciones distinto, y de hecho este girará en torno a "clases de filósofos" y no a "posteridades críticas",

9 Michel Foucault, Le gouvernement de soi et des autres: Cours au Collège de France, 1982-1983 (París: Éditions Gallimard, 2008): 22.

10 Frédéric Gros, "Situation du Cours", en Le gouvernement de soi et des autres, Cours au Collège de France, 1982-1983, Michel Foucault (París: Éditions Gallimard, 2008): 347-361. 
el pensador francés reivindicará posnietzscheanamente la ligazón entre arqueología (modo en que, por entonces, denominaba su trabajo) y diagnóstico:

A propósito de Nietzsche, podemos volver sobre su pregunta: para él, el filósofo es quien diagnostica el estado del pensamiento. Por cierto, se pueden concebir dos clases de filósofos, el que abre nuevos caminos al pensamiento, como Heidegger, y el que juega en cierta forma el rol de arqueólogo, que estudia el espacio en el que se despliega el pensamiento, así como sus condiciones, su modo de constitución. ${ }^{11}$

Enfatizamos, entonces, que Foucault destacaba tempranamente (siguiendo la estela de Nietzsche) el objetivo de carácter diagnóstico que atravesaba sus trabajos. Por ende, no es aventurado revisar sus indagaciones recurrentes respecto de las ciencias humanas destacando la manera en que el trabajo de archivo sobre tales discursos se encuentra motorizado por preocupaciones ontológico-políticas respecto de la constitución de la actualidad. En la misma dirección, no puede pasarse por alto que, también en el contexto de la publicación de su arqueología de 1966, Foucault brindaría una entrevista en la que se mostraría elogioso con respecto al estructuralismo, en tanto, según su lectura, el estructuralismo permitiría diagnosticar qué es la actualidad. ${ }^{12}$ A mayor abundamiento, no podemos dejar de remarcar que, en la efervescencia suscitada por la publicación de este libro, el filósofo responderá a una reseña crítica, enfatizando la relevancia de pensar una política progresista no humanista. ${ }^{13}$

A partir de la lectura que hemos desplegado en los párrafos precedentes, sostenemos que, más allá de los matices, las declinaciones y las tensiones que puedan reseñarse, la problematización de la filosofía como una actividad de diagnóstico crítico del presente atraviesa la producción foucaultiana. Por tanto, consideramos que en tanto sus lecturas recurrentes sobre el discurso de las ciencias humanas (en las que nos detendremos a continuación) se inscriben en esta forma de problematización del trabajo filosóficocrítico, se erigen a partir de una serie de indagaciones en que la crítica epistemológica desplegada en torno al trabajo de archivo no puede considerarse como un fin en sí mismo, sino que, por el contrario, resulta palpable que se encuentra jalonada por la preocupación ontológico-política de diagnosticar el presente. En torno a lo cual cabe recordar que, en sus arqueologías publicadas durante la primera mitad de la década de 1960 acerca de la locura ${ }^{14}$ y de la clínica, ${ }^{15}$ Foucault problematiza la imbricación entre la formación de determinados saberes, vinculados a la constitución del "hombre" como objeto, ${ }^{16}$ y las transformaciones en las prácticas institucionales, en consonancia con sus posteriores indagaciones genealógicas desarrolladas en la década de $1970 .{ }^{17}$ Sin embargo, para disipar objeciones, se nos impone

11 Michel Foucault, “Qu’est-ce qu'un philosophe?”, en Dits et écrits, 1954-1988. IV. I. 1954-1969 (París: Éditions Gallimard, 1994): 553.

12 Michel Foucault, "La philosophie structuraliste permet de diagnostiquer ce qu'est 'aujourd'hui”, en Dits et écrits, 1954-1988. IV. I. 1954-1969 (París: Éditions Gallimard, 1994): 580-584.

13 Michel Foucault, "Réponse à une question", en Dits et écrits. 1954-1988. I. 1954-1969 (París: Éditions Gallimard, 1994): 673-695.

$14 \mathrm{Si}$ bien remitimos a la segunda edición, para evitar confusiones recordamos que la primera fue presentada como tesis doctoral en 1961. Michel Foucault, Histoire de la folie à l’âge classique (París: Éditions Gallimard, 1972).

15 Michel Foucault, Naissance de la clinique: Une archéologie du regard médical (París: Presses universitaires de France, 1963).

16 Philippe Sabot, Lire Les mots et les choses de Michel Foucault (París: Presses universitaires de France, 2006 ): 60.

17 Gary Gutting, Michel Foucault's archaeology of scientific reason (Cambridge: Cambridge University Press, 1989): 109-110. 
la necesidad de introducir la siguiente aclaración terminológica. Si bien por "ciencias humanas" Foucault alude a los estudios literarios, la sociología y las ciencias con radical "psi”, en la medida en que remarca que estas se configuraron a partir del plexo formado por las ciencias respecto de la vida, el trabajo y el lenguaje, para simplificar la redacción, nos valemos de la fórmula "ciencias humanas" o "saberes acerca de lo humano" en sentido amplio y englobante, para aludir a la disposición epistémica en que dichas ciencias se formaron. ${ }^{18}$ Por ese motivo, previamente aludimos a la arqueología de la constitución del objeto locura como "enfermedad mental" y al trabajo arqueológico abocado a dar cuenta del nacimiento de la mirada médica, en tanto se vinculan a la constitución del "hombre" como objeto de saber que vive, trabaja y habla.

Ahora bien, sin desconocer que la problematización de la imbricación entre las prácticas discursivas y las extradiscursivas parece elidida en Les mots et les choses, la preocupación ontológico-política (unida al diagnóstico del presente) que atraviesa tal arqueología resulta, sin embargo, insoslayable, puesto que de lo que allí se trata es de problematizar la constitución del "hombre", de modo tal de poder desasirse de la grilla de inteligibilidad forjada por el humanismo, que hace de esta figura el punto de partida tanto de la reflexión teórico-epistemológica como de la indagación práctico-política. Además, es de destacar que, tras trazar la arqueología de las ciencias humanas, Foucault les contrapondrá las configuraciones epistemológicas articuladas en torno al encuadre estructuralista, caracterizando la etnología y el psicoanálisis como "contraciencias humanas". En este contexto, consideramos más que atinada la interpretación propuesta por Luca Paltrinieri, quien respecto de la vinculación entre la indagación arqueológica y la preocupación por el diagnóstico crítico de la actualidad sostuvo que, "si la historia posee un privilegio en la óptica foucaultiana, es precisamente porque permite abstraerse del propio presente, tomar distancia de la propia cultura cotidiana para examinar 'desde el exterior' nuestro sistema de certezas y discursos". ${ }^{19}$

Finalmente, querríamos destacar que el abordaje recurrente del discurso de las ciencias humanas no se circunscribe a los trabajos desarrollados durante la década de 1960, sino que atraviesa las grandes genealogías de la década de 1970. Por ejemplo, en la genealogía de la prisión como dispositivo disciplinario, se destaca la imbricación entre el surgimiento del dispositivo y la configuración de saberes acerca de "lo humano" como la criminología ${ }^{20}$ relación que resulta fundamental también en la genealogía del dispositivo de sexualidad. ${ }^{21}$ Del mismo modo, cabe recordar que en sus cursos dedicados al estudio del surgimiento de la biopolítica, ${ }^{22}$ de los dispositivos de seguridad ${ }^{23}$ y del liberalismo y el neoliberalismo en tanto racionalidades de gobierno, ${ }^{24}$ el trabajo de archivo sobre los saberes acerca de "lo humano" resulta central, sea bajo la figura del hombre como ser viviente, como hablante o como trabajador. Específicamente, en la clase del 25 de enero de 1978, correspondiente al curso Sécurité,

18 Michel Foucault, Les mots et les choses: Une archéologie des sciences humaines (París: Éditions Gallimard, 1966).

19 Luca Paltrinieri, “Archeologia della volontà: Una preistoria delle Lezioni sulla volontà di sapere”, Quadranti: Rivista Internazionale di Filosofia Contemporanea 2, n. 1 (2014): 108.

20 Michel Foucault, Surveiller et punir: Naissance de la prison (París: Éditions Gallimard, 1975); Guillaume Le Blanc, La pensée Foucault (París: Ellipses Édition, 2014): 135-149.

21 Michel Foucault, La volonté de savoir: Histoire de la sexualité 1 (París: Éditions Gallimard, 1976).

22 Michel Foucault, "Il faut défendre la société": Cours au Collège de France, 1976 (París: Éditions Gallimard, 1997).

23 Michel Foucault, Sécurité, territoire, population: Cours au Collège de France, 1977-78 (París: Éditions Gallimard, 2004).

24 Michel Foucault, Naissance de la biopolitique: Cours au Collège de France, 1978-79 (París: Éditions Gallimard, 2004). 
territorie, population, reformuló la mutación epistemológica aborda doce años antes en Les mots et les choses, al señalar que la formación de las ciencias de la vida, el trabajo y el lenguaje, en cuyo plexo se configuraron las ciencias humanas, tuvo lugar en el acontecimiento de surgimiento de los dispositivos de seguridad, conexos a la formación de la población como blanco privilegiado y la configuración de la economía política como saber estratégico respecto de cómo gobernar. ${ }^{25}$ En dicha clase, destacará Foucault que el hombre surge como "figura de la población".

\section{Arqueo-genealogía y diagnóstico: un método de trabajo filosófico en las canteras de la historia orientado a la interrogación del presente}

La arqueología como método, y específicamente Las palabras y las cosas, son una propedéutica a la genealogía. La genealogía, tal como nos fue presentada por Foucault, no es la crisis de la arqueología, sino que ellas se apuntalan mutuamente. ${ }^{26}$

A partir de la reconstrucción propuesta en el apartado precedente, por medio de la que buscamos brindar un perfil de la filosofía foucaultiana, al detenernos en su caracterización de la crítica como actividad de diagnóstico del presente y destacar la centralidad que posee el discurso de las ciencias humanas en el recurrente trabajo de archivo desplegado por el filósofo en pos de elaborar un diagnóstico crítico del presente, en este apartado nos ocuparemos de reponer las "cuestiones de método" en que Foucault se apoya al llevar a cabo el mencionado trabajo de archivo. En consonancia con la cita de Daniel Defert que hemos puesto como epígrafe, que, ciertamente, se hace eco de la referencia que hemos puesto del trabajo de Sven-Olov Wallenstein al comienzo de este artículo, en lugar de retomar las clásicas lecturas etapistas, y como contracara de ello de reconstruir "el método arqueológico" y "el método genealógico", nos ocuparemos de reponer la problematización arqueológica del método de trabajo filosófico (desplegado en las canteras de la historia y jalonado por el objetivo ontológico-político de contribuir al diagnóstico del presente), tras lo cual revisaremos lo que damos en llamar "ecos arqueológicos de la genealogía”, y así perfilar una forma de problematización fruto de la reformulación y reelaboración recurrente que resulta plausible denominar arqueo-genealogía foucaultiana.

Si bien hemos aludido previamente de modo parcial a los que podrían denominarse "ecos arqueológicos de la genealogía", en tanto hemos señalado el arco crítico en que se inscribe el corpus foucaultiano, y en este contexto hemos reseñado la manera recurrente en que Foucault vuelve sobre el discurso de las ciencias humanas al desplegar el trabajo de archivo en sus grandes genealogías, en este apartado, al revisar las "cuestiones de método", nos detendremos en la centralidad que la noción de saber conserva en sus reflexiones acerca del método genealógico. Por otra parte, antes de adentrarnos en la problematización arqueológica del método, consideramos pertinente recordar que hacia el final de Lachéologie du savoir Foucault explicitó la posibilidad de llevar a cabo "otras arqueologías", es decir, trabajos arqueológicos

25 Santiago Castro-Gómez, Historia de la gubernamentalidad: Razón de Estado, liberalismo y neoliberalismo en Michel Foucault (Bogotá: Siglo del Hombre, 2010): 16; Emiliano Sacchi, "Umbrales biológicos de la modernidad política en Michel Foucault”, Daimon: Revista Internacional de Filosofía, n. 68 (2016): 32-33.

26 Daniel Defert, "Situation du Cours", en Leçons sur la volonté de savoir: Cours au Collège de France, 1970-71, Michel Foucault (París: Éditions Gallimard, 2011): 271. 
que no se ciñeran fundamentalmente al discurso de los saberes, sino que se abocaran, por ejemplo, al estudio de la pintura, la sexualidad y la política. Justamente, la sexualidad y la política se convertirán en blancos privilegiados a lo largo de sus investigaciones desarrolladas durante la década de $1970,{ }^{27}$ en el contexto del relanzamiento de su trabajo hacia una morfología de la voluntad de saber, problematizada en términos metodológicos desde la perspectiva de una genealogía de las relaciones de saber-poder. Proyecto que recorrerá los cursos dictados y los libros publicados a lo largo de la década de 1970, y que será reelaborado en el curso dictado a comienzos de 1980, en el que reformulará la grilla saber-poder respecto del gobierno mediante la verdad y problematizará el método como una anarqueología de las prácticas aletúrgicas. ${ }^{28}$

Ahora bien, en función de los objetivos propuestos, dirigimos la lectura, en primer lugar, hacia el modo en que Foucault problematiza arqueológicamente el método de trabajo filosófico a finales de la década de 1960, cuando se dispone a "pasar en limpio" el método arqueológico y sus supuestos epistemológicos, a partir de una relectura de sus investigaciones precedentes. En ese sentido, sostuvo el filósofo que la problematización arqueológica de los discursos consiste en abordarlos en tanto prácticas y se orienta a dar cuenta de sus condiciones de posibilidad, y así lograr el establecimiento de los modos históricos de constitución de ciertas positividades, en lugar de tomarlas de antemano como evidencia y punto de partida. ${ }^{29}$ En sus propios términos:

Pero de lo que aquí se trata no es de neutralizar el discurso, de hacerlo el signo de otra cosa y de atravesar su espesor para alcanzar aquello que permanece silenciosamente más allá de él, sino, al contrario, de mantenerlo en su consistencia, de hacerlo surgir en la complejidad que le es propia [...]. Sustituir el tesoro enigmático de las "cosas" anteriores al discurso, por la formación regular de los objetos que no se perfilan más que en él. Definir esos objetos sin referencia al fondo de las cosas, sino en relación al conjunto de las reglas que permiten formarlos como objetos de un discurso y constituyen así sus condiciones de aparición histórica. ${ }^{30}$

De lo que se trata, entonces, es de una forma de problematización de los discursos en tanto prácticas que se ocupa de problematizar los enunciados y rastrear las reglas que regulan el ejercicio de la función enunciativa. De este modo, en lugar de "hacer pie" en el sujeto (fundador) o en el objeto (anterior al discurso), el pensador francés ubica el registro arqueológico en una posición de exterioridad con respecto a los discursos que problematiza y se ocupa de desentrañar las reglas que regulan el ejercicio de la función enunciativa y constituyen de manera inmanente las modalidades enunciativas, los objetos, las elecciones temáticas y los conceptos. No puede pasarse por alto que el pasaje de la problematización de

27 Edgardo Castro, Lecturas foucaulteanas: Una historia conceptual de la biopolítica (Buenos Aires: UNIPE Editorial Universitaria, 2011): 43-45; Pablo Martín Méndez, "Foucault y la arqueología de la política: Tras las huellas de un método inconcluso", Revista de Filosofía Diánoia 65, n. ${ }^{\circ} 84$ (2020): 81-109.

28 Michel Foucault, Du gouvernement des vivants: Cours au Collège de France, 1979-80 (París: Éditions Gallimard, 2012); Julián Sauquillo González, "La 'anarqueología' como crítica del gobierno de los hombres por la verdad", en Michel Foucault: Derecho y poder, ed. por Mauro Benente (Buenos Aires: Didot, 2015): 67-90; Maite Larrauri, Anarqueología: Foucault y la verdad como campo de batalla (Madrid: Enclave de Libros, 2018).

29 Alain Brossat, "L'archive et les archives: Archéologie des discours et gouvernement des vivants", Materiali Foucaultiani 2, n. $^{\circ} 4$ (2013): 238-239.

30 Michel Foucault, Larchéologie du savoir (París: Éditions Gallimard, 1969): 65. 
los objetos y las modalidades enunciativas hacia sus reglas de formación resulta un aporte epistemológico fundamental, puesto que permite desanclar la epistemología de las ciencias humanas del interior de la relación sujeto-objeto y visibilizar los términos de la relación cognoscitiva como inmanentes a tales reglas de formación. Este modo de problematización de los saberes en tanto prácticas discursivas, que pone como blanco privilegiado del trabajo de archivo las formaciones discursivas, ${ }^{31}$ permitió a Foucault plantear la oposición entre la teoría del conocimiento, que desde su perspectiva se ocuparía de dar cuenta de la relación sujeto-objeto, y la arqueología del saber que se pone por fuera de esta relación, y permite desbrozar la formación de ambos términos en tanto inmanentes al ejercicio de la función enunciativa. Al respecto, el filósofo sostuvo:

El enunciado no es, entonces, una estructura (es decir, un conjunto de relaciones entre elementos variables, que autorizaría un número quizás infinito de modelos concretos); es una función de existencia que pertenece en propiedad a los signos y a partir de la cual se puede decidir, por lo tanto, por medio del análisis o la intuición, si "tienen sentido" o no, según qué reglas se suceden o se yuxtaponen, de qué son signo, y qué suerte de acto se encuentra efectuado por su formulación (oral o escrita). No hay que sorprenderse si no se han podido encontrar criterios estructurales de unidad para el enunciado; puesto que no es en sí mismo una unidad, sino una función que cruza un dominio de estructuras y unidades posibles y las hace aparecer, con contenidos concretos, en el tiempo y el espacio. ${ }^{32}$

A partir de la puesta en cuestión de las unidades de discurso comúnmente aceptadas en el ámbito de la denominada "historia de las ideas", es decir, de la realización de un trabajo negativo que implica la puesta entre paréntesis de nociones tales como tradición, libro, autor, mentalidad, espíritu de época, el trabajo epistemológico a realizar se desplegará según la labor paciente y sistemática sobre documentos, a los que se tratará como monumentos. Por tanto, la puesta en práctica de la arqueología trae aparejada la realización de un trabajo archivístico sobre los discursos que, al abordarlos en tanto prácticas, dé cuenta de sus condiciones de posibilidad ${ }^{33}$ al desentrañar la configuración del denominado a priori histórico. En términos del autor:

Yuxtapuestos, estos dos términos hacen un efecto un poco resonante; entiendo designar con ello un a priori que no sería condición de validez para unos juicios, sino condición de realidad para unos enunciados. No se trata de encontrar aquello que podría hacer legítima una aserción, sino de aislar las condiciones de emergencia de unos enunciados, la ley de su coexistencia con otros, la forma específica de su modo de ser, los principios según los cuales ellos subsisten, se transforman y desaparecen. ${ }^{34}$

En un debate con el intelectual italiano Giulio Preti, realizado en 1972, Foucault se encargaría de explicitar de manera rotunda la radical incompatibilidad entre la noción de a priori histórico, como parte del acervo de herramientas que constituyen el método arqueológico, y una reflexión antropológico-gnoseológica de corte kantiano dirigida a la búsqueda de categorías universales constitutivas del sujeto trascendental y, por tanto, constituyentes de la experiencia:

31 Foucault, L’archéologie du savoir, 53.

32 Foucault, L’archéologie du savoir, 115.

33 Edgardo Castro, Pensar a Foucault: Interrogantes filosóficos de La arqueología del saber (Buenos Aires: Biblos, 1995): 39.

34 Foucault, L’archéologie du savoir, 167. 
Lo que he llamado "episteme" en Las palabras y las cosas, no tiene nada que ver con categorías históricas; es decir, en suma, con categorías que fueron creadas en un determinado momento histórico. Cuando hablo de episteme, me refiero a todas las relaciones que existieron en una determinada época entre los diferentes dominios de la ciencia. [...] Por lo tanto, para mí, la episteme no tiene nada que ver con las categorías kantianas [...]. No soy kantiano ni cartesiano, precisamente, porque rechazo una identificación a nivel trascendental entre sujeto y yo pensante. Estoy seguro de que existen, si no estructuras, propiamente hablando, reglas de funcionamiento del conocimiento que aparecieron en el curso de la historia y al interior de las cuales se sitúan los diferentes sujetos. ${ }^{35}$

Recapitulando la lectura que hemos venido desarrollando, sostenemos que el eje de la apuesta foucaultiana radica en la problematización del saber como un modo alternativo a la elaboración de una teoría del conocimiento, puesto que no se trata, para Foucault, de problematizar los términos de la relación cognoscitiva, sino de ubicarse por fuera de la relación misma, dando lugar al problema del saber en oposición a la del conocimiento, ${ }^{36}$ en la medida en que la arqueología se despliega por medio del abordaje de la historia efectiva de las prácticas discursivas. En ese sentido, permite llevar a cabo una indagación que no se encuentra motorizada por preocupaciones normativas, al buscar dar cuenta de las condiciones de existencia de los discursos científicos en lugar de interrogarse respecto de qué es lo que daría derecho a un discurso a ser ciencia, ${ }^{37}$ y que se realiza por medio del trabajo de archivo que prescinde de tomar la ciencia actual como vara para la realización de una historia en clave teleológica:

No se interrogarán los conocimientos descritos en su progreso hacia una objetividad en la que la ciencia actual pudiera reconocerse; lo que se pondrá en juego es el campo epistemológico, la episteme en que los conocimientos, vistos más allá de todo criterio referente a su valor racional o a sus formas objetivas, hunden su positividad y manifiestan así una historia que no es la de su perfección creciente, sino más bien la de sus condiciones de posibilidad. ${ }^{38}$

Tal como lo señaláramos, la problematización de las ciencias humanas en tanto saberes se ejercita de manera desligada respecto de la dupla formada por el par enfoque teleológico-pretensiones normativas. Es decir que la arqueología se ocupa de interrogar los saberes en su historia efectiva, para lo que prescinde de tomar como punto de partida filosófico la ciencia actual y de hacerla funcionar como punto de llegada necesario de la historia de la ciencia, al tiempo que dicha interrogación no se encuentra jalonada por la búsqueda de criterios que permitan regular la puesta en práctica de la ciencia. Ahora bien, retornando al interrogante con el que abriéramos este artículo, sostenemos que en la distinción entre saber y conocimiento, que habilita el desanclaje de la epistemología de las ciencias humanas del interior de la relación sujeto-objeto, la perspectiva arqueológica permite elaborar un registro de problematización que prescinde de tomar la ciencia actual historiográficamente en términos teleológicos y epistemológicamente de modo normativo, y da lugar a un trabajo epistemológico que en vez de "bastarse a sí mismo" se perfila como una herramienta potente para la problematización ontológico-política de la actualidad.

35 Michel Foucault, "Les problèmes de la culture, un débat Foucault-Preti”, en Dits et écrits, 1954-1988. II. 1970-1975 (París: Éditions Gallimard, 1994): 371-373.

36 Michel Foucault, “Sur l’archéologie des sciences: Réponse au Cercle d’épistémologie”, en Dits et écrits, 1954-1988. I. 1954-1969 (París: Éditions Gallimard, 1994): 730.

37 Foucault, Larchéologie du savoir, 249-251.

38 Michel Foucault, Les mots et les choses: Une archéologie des sciences humaines (París: Éditions Gallimard, 1966$): 13$. 
La problematización del saber permite dar cuenta del modo en que históricamente (de manera inmanente a cierta disposición epistémica) se constituyen objetos y posiciones de sujeto. Por tanto, en lugar de brindar herramientas para la realización de una crítica normativa respecto de la "objetividad cognoscitiva" de las ciencias humanas, la crítica arqueológica se desplaza hacia la problematización de las formas de objetivación. Consideramos, entonces, que la arqueología foucaultiana del saber no se encuentra jalonada por las preguntas propias de la "analítica de la verdad en general", es decir, de las posibilidades del conocimiento y sus límites infranqueables, sino que, por el contrario, al brindar herramientas para la problematización de los modos históricos de constitución de objetos y posiciones de sujeto (modalidades enunciativas) contribuye a la práctica de la crítica entendida como actividad de diagnóstico de la actualidad. Las indagaciones epistemológicas del arqueólogo se encuentran jalonadas por preocupaciones de índole ontológico-política, de ahí que, en lugar de intentar dar cuenta del clásico problema (de cuño normativo) que ha permeado el discurso epistemológico de las ciencias humanas, es decir, la pregunta respecto de su "objetividad cognoscitiva", sostenemos que Foucault se desmarca de este registro discursivo, desplazando la pregunta hacia la problematización de las formas de objetivación. Ahora bien, hemos dado inicio al presente apartado señalando "ecos arqueológicos de la genealogía". A esta altura, podríamos agregar, para proseguir con la lectura, que Foucault presenta la genealogía como un método de trabajo filosófico que se despliega en las canteras de la historia y que prescinde de adoptar una perspectiva crítica articulada por el par "teleología-normación”, en torno a lo que conserva un lugar central la contraposición entre saber y conocimiento. En ese sentido, en la "Leçon sur Nietzsche", dictada por Foucault en Montreal en 1971, problematiza, a partir de una lectura de Nietzsche, la posibilidad de trazar "una historia de la verdad que no se apoye en la verdad". Historia de la verdad a la que presenta en oposición a la elaborada en la filosofía positivista comteana:

En esta historia positivista, la verdad no está dada al comienzo. Durante mucho tiempo, el conocimiento la busca: ciego, titubeante. La verdad se da como el resultado de una historia. Pero esa relación finalmente establecida entre la verdad y el conocimiento es una relación de derecho que se plantea al comienzo. El conocimiento está hecho para ser conocimiento de la verdad. Hay una copertenencia de origen entre la verdad y el conocimiento. [...] El atrevimiento de Nietzsche consiste en haber desanudado esas implicaciones. Y haber dicho: la verdad sobreviene al conocimiento sin que el conocimiento esté destinado a la verdad, sin que ella sea la esencia del conocer. [...] Pensar el conocimiento como un proceso histórico anterior a todo problema de la verdad, y más fundamentalmente que en la relación sujeto-objeto. El conocimiento liberado de la relación sujeto-objeto es el saber. ${ }^{39}$

Sostenemos, entonces, que la problematización genealógica recupera los desarrollos arqueológicos, puesto que la "des-implicación" entre conocimiento y verdad, que se relaciona con la posibilidad de repensar el conocimiento respecto del saber, es decir, de pensarlo por fuera de la relación sujeto-objeto, retoma la forma de problematización forjada por Foucault en la contraposición entre teoría del conocimiento y arqueología del saber. Cabe remarcar que en la cita precedente el filósofo vincula la noción de que el saber no se da entre sujeto y objeto, sino que ambos términos le son inmanentes, al hecho de que la interrogación se genera por fuera del problema de la verdad, por fuera de un problema pensable en la relación cognoscitiva. De este modo, en su lectura de Nietzsche, Foucault destaca la posibilidad de llevar a cabo una historia de la verdad en la que no entren en juego las verdades inmanentes a la

39 Michel Foucault, "Leçon sur Nietzsche”, en Leçons sur la volonté de savoir: Cours au Collège de France, 1970-71 (París: Éditions Gallimard, 2011): 199-205. 
formación discursiva de los saberes contemporáneos de modo normativo, tanto epistemológica como historiográficamente. En términos del pensador francés:

Las investigaciones realizadas previamente permitieron reconocer un nivel singular entre aquellos que hacen posible analizar los sistemas de pensamiento: el de las prácticas discursivas. Se trata de una sistematicidad que no es de tipo lógico ni lingüístico. Las prácticas discursivas se caracterizan por el recorte de un campo de objetos, la definición de una perspectiva legítima para el sujeto de conocimiento, la fijación de normas para la elaboración de conceptos y teorías. Cada una de ellas supone, entonces, un juego de prescripciones que rigen las exclusiones y elecciones. [...] Los caracteres generales de esas prácticas y los métodos propios para analizarlas se inventariaron bajo el nombre de arqueología. ${ }^{40}$

El desplazamiento arqueológico desde el conocimiento hacia el saber habilita una forma de problematización que se erige por fuera del problema de la verdad y, por ende, queda al abrigo de valerse del "conocimiento actual" en términos historiográficamente teleológicos y epistemológicamente normativos, es decir, de apoyarse en la verdad a la hora de indagar su historia. Por otra parte, en 1971, Michel Foucault publica "Nietzsche, la généalogie, l'histoire", artículo en el que, a través de una lectura de Nietzsche, retoma la distinción entre Ursprung y Erfindung, términos alemanes que implican la noción de origen e invención, respectivamente. Por tanto, en la lectura foucaultiana de Nietzsche, Ursprung es vinculado con la noción metafísica de origen fuente, de origen transhistórico, mientras Erfindung se refiere al problema concreto de la procedencia (Herkunft) y de las condiciones de posibilidad para la emergencia o surgimiento (Entstehung) de las prácticas. En términos de Foucault: "la procedencia permite reencontrar bajo el aspecto único de un carácter, o de un concepto, la proliferación de los acontecimientos a través de los cuales (gracias a los cuales, contra los cuales) ellos se formaron". ${ }^{41}$ Hacia el final del mismo párrafo, nuestro "autor" agregará entonces:

No hay nada que se parezca a la evolución de una especie, al destino de un pueblo. Seguir la filial compleja de la procedencia es, por el contrario, mantener lo que pasó en la dispersión que le es propia [...]; es descubrir que en la raíz de lo que conocemos y de lo que somos no están en absoluto la verdad y el ser, sino la exterioridad del accidente. ${ }^{42}$

Encontramos allí la explicitación foucaultiana de la cantera abierta por el abandono de una concepción teleológica de las prácticas, cuya contrapartida no es ni más ni menos que la puesta en cuestión del carácter "evidente" de universales como verdad y ser. Por otro lado, sería pertinente destacar que, en la lectura de Nietzsche propuesta por Foucault, el análisis de la emergencia, es decir, la indagación respecto de las denominadas condiciones de posibilidad de esta, constituye una herramienta que posibilita la introducción de las fuerzas y sus correlaciones en el abordaje genealógico de los problemas filosóficos. Tal como lo explicitara en este artículo:

40 Michel Foucault, "Résumé du Cours", en Leçons sur la volonté de savoir: Cours au Collège de France, 1970-71 (París: Éditions Gallimard, 2011): 217-218.

41 Michel Foucault, “Nietzsche, la généalogie, l’histoire”, en Dits et écrits, 1954-1988. II. 1970-1975 (París: Éditions Gallimard, 1994): 141.

42 Michel Foucault, "Nietzsche, la généalogie, l'histoire”, en Dits et écrits, 1954-1988. II. 1970-1975 (París: Éditions Gallimard, 1994): 141. 
Entstehung designa más bien la emergencia, el punto de surgimiento. Es el principio y la ley singular de una aparición [...]. La genealogía restablece los diversos sistemas de avasallamiento: no la potencia anticipadora de un sentido, sino más bien el juego azaroso de las dominaciones. [...] La emergencia se produce siempre en un cierto estado de fuerzas. ${ }^{43}$

No se trata, entonces, de indagar las prácticas desde una teleología de la anticipación, en el caso particular de los saberes: desde la perspectiva del progreso paulatino de la historia de la ciencia desde el error hacia la verdad, sino de la interrogación de estos en su contingencia, es decir, en el azar de las luchas y dominaciones. Dos años más tarde, Foucault dictará, en Río de Janeiro, el ciclo de conferencias La vérité et les formes juridiques; en la primera de ellas, se detendría nuevamente en la exposición de su lectura de la genealogía nietzscheana. Allí destacará que las herramientas nietzscheanas habilitan la realización de una historia de la verdad descargada de un enfoque teleológico y normativo. ${ }^{44}$ Hacia finales de la década de 1970, introduce la noción de crítica política del saber, que es presentada, en contraposición a la crítica de la ideología, en las clases del 10 y del 17 de enero de 1979, con las que diera inicio al curso Naissance de la biopolitique. Allí, al explicitar las "cuestiones de método" que vertebran la problematización desplegada a lo largo del curso, dedicado al estudio de la formación de la economía política y del surgimiento del liberalismo como prisma reflexivo gubernamental, el filósofo planteó:

La crítica que les propongo consiste en determinar bajo qué condiciones y con qué efectos se ejerce una veridicción, es decir, una vez más, un tipo de formulación dependiente de ciertas reglas de verificación y falseamiento. [...] No es la historia de lo verdadero, no es la historia de lo falso, es la historia de la veridicción la que posee importancia política. ${ }^{45}$

Frente a la puesta en cuestión del discurso científico decimonónico en términos de "conocimiento superado" por la ciencia actual, cuyo carácter político se relacionaría con el hecho de que se trataba de "conocimiento todavía no suficientemente elaborado", "contaminado ideológicamente", que tendría por presunta función la "legitimación del poder", la crítica política del saber llevada a cabo por el genealogista permite dar cuenta del modo en que en determinado momento histórico se produjo un acoplamiento entre una serie de prácticas y un régimen de veridicción. Tal como se desprende de la palabra foucaultiana, la problematización de los saberes apuntará a desbrozar la manera en que estos articularon una serie de prácticas, constituyendo ciertos objetos pasibles de ser interrogados a partir de determinadas reglas de verificación y falseamiento. Es decir que de lo que se trata, a la hora de llevar a cabo una crítica política del saber, es ni más ni menos que de la realización de una crítica de las formas de objetivación inmanentes a determinado régimen de veridicción. Crítica epistemológica articulada por una preocupación ontológica-política, es decir, referida al ejercicio de la filosofía como actividad de diagnóstico, que permite indagar la formación inmanente de los objetos y de las modalidades enunciativas que configuran las posiciones de sujeto, esto es, cómo se constituye quién habla y de qué habla en determinado régimen de veridicción. En lugar de brindar herramientas para la realización de una crítica normativa respecto de la "objetividad cognoscitiva" de las ciencias humanas, la crítica política del saber se ubica en el registro configurado a partir del desplazamiento hacia la puesta en cuestión de las

43 Foucault, "Nietzsche, la généalogie, l'histoire”, 143.

44 Michel Foucault, "La vérité et les formes juridiques", en Dits et écrits, 1954-1988. II. 1970-1975 (París: Éditions Gallimard, 1994): 538-646.

45 Michel Foucault, Naissance de la biopolitique: Cours au Collège, 1978-79 (París: Éditions Gallimard, 2004): 37-38. 
formas de objetivación erigido por la arqueología. Abordaje de los saberes acerca de "lo humano" que, en lugar de cuestionar su "falta de objetividad" y contraponerles un "modo adecuado de conocer", se ejercita por medio de la problematización acerca de la formación de tales objetos. Sostenemos, entonces, que el método arqueogenealógico, articulado en torno a la propuesta de llevar a cabo una crítica política del saber, contribuye al diagnóstico de la actualidad por medio de la realización de una crítica de las formas de objetivación.

\section{Consideraciones finales}

Foucault se propone mostrar cómo las prácticas sociales pueden llegar a engendrar dominios de saber que no solo hacen que aparezcan nuevos objetos, conceptos y técnicas, sino que hacen nacer, además, formas totalmente nuevas de sujetos y sujetos de conocimiento. En este sentido, Foucault afirma que el sujeto de conocimiento posee una historia: la relación del sujeto con el objeto, y más claramente: la verdad misma tiene una historia. ${ }^{46}$

En vista de realizar una contribución a este dosier temático, a lo largo de nuestro artículo nos hemos ocupado de reconstruir la manera en que el filósofo francés Michel Foucault problematiza las herramientas de trabajo filosófico en su concepción del ejercicio de la crítica como actividad de diagnóstico del presente. En primer lugar, nos detuvimos en brindar un perfil de la filosofía foucaultiana tomando como punto de partida el característico gesto del filósofo, consistente en la recuperación y reelaboración recurrente de sus trabajos precedentes desde la perspectiva de sus indagaciones en curso. En ese sentido, nos ocupamos de enfatizar el modo en que Foucault problematiza sus trabajos a partir de una caracterización de la filosofía como actividad de diagnóstico del presente, en un arco que se extiende (al menos) desde 1966 hasta su muerte en 1984, en torno a lo cual nos hemos detenido específicamente en la forma en que se ubica respecto de la contraposición entre "clases de filósofos" en 1966 y en una de las "posteridades críticas" en 1983. Complementariamente, dado que esta revisión la hemos realizado como antesala de la reconstrucción de las "cuestiones de método", nos hemos ocupado, justamente, de reponer el abordaje recurrente del discurso de las ciencias humanas realizado por Foucault, en tanto en cuanto constituyen un blanco privilegiado de su trabajo de archivo abocado al diagnóstico del presente. A continuación, en el siguiente apartado, nos ocupamos de reconstruir la problematización arqueológica del método de trabajo filosófico elaborada por Foucault a finales de la década de 1960, deteniéndonos particularmente en L'archéologie du savoir, libro en que el filósofo se propone "pasar en limpio" el método arqueológico y sus supuestos epistemológicos a partir de la relectura crítica de sus investigaciones precedentes. Específicamente, nos detuvimos en su problematización de las prácticas discursivas, destacando que este modo de abordaje introduce un viraje desde la teoría del conocimiento, al desanclar la epistemología de las ciencias humanas del interior de la relación sujeto-objeto y ocuparse de dar cuenta de la formación inmanente de las posiciones de sujeto (modalidades enunciativas) y la constitución de los objetos. A partir de este viraje, la arqueología del saber se desmarca de un registro normativo de interrogación de las ciencias humanas y se propone dar cuenta, por el contrario, de sus condiciones de posibilidad al desentrañar las reglas que regulan el ejercicio de la función enunciativa que, en el

46 Marcelo Raffin, "Natureza e política: Uma tensão no pensamento de Michel Foucault", Ágora Filosófica 1, n. 2 (2014): 130. 
seno de determinado a priori histórico, dieron lugar a la constitución de la vida, el trabajo, el lenguaje y "lo humano" en tanto objetos de saber, con la correlativa constitución de las modalidades enunciativas correspondientes. Esta forma de ejercicio de la crítica, que se desmarca de una perspectiva fundadoranormativa, y que apunta hacia el diagnóstico ontológico-político de la constitución del presente, no realiza un aporte al perenne problema de la objetividad cognoscitiva de las ciencias humanas (de cuño normativo), sino que, por el contrario, habilita un modo de crítica que hemos denominado "crítica de las formas de objetivación". Crítica epistemológica que, remarcamos una vez más, no se "basta a sí misma", sino que contribuye al diagnóstico ontológico-político del presente.

Luego, pusimos el foco de mira en la problematización genealógica del método, al ocuparnos de reponer las reflexiones foucaultianas acerca de su modo de desplegar el trabajo de diagnóstico crítico del presente al dirigir la mirada a las canteras de la historia, tomando como herramienta una lectura de los trabajos de Nietzsche. Frente a las clásicas lecturas "etapistas", revisamos estos trabajos elaborados por Foucault a comienzos de la década de 1970, destacando lo que denominamos "ecos arqueológicos de la genealogía", tras lo cual nos dirigimos hacia las "cuestiones de método" que el filósofo introdujo en 1979, cuando propuso llevar adelante una crítica política del saber, propuesta que, sostenemos, articula la problematización foucaultiana del método de trabajo filosófico en una perspectiva que, habida cuenta de los ecos arqueológicos que la habitan, designamos "arqueogenealógica". A modo de cierre, enfatizamos, entonces, que la crítica política del saber posibilita la puesta en cuestión de la manera en que históricamente, de forma inmanente a cierta disposición epistémica, se constituyen objetos y posiciones de sujeto. Por tanto, en lugar de brindar herramientas para la realización de una crítica normativa respecto de la "objetividad cognoscitiva" de las ciencias humanas, la crítica política del saber se ubica en el seno del desplazamiento operado por la arqueología hacia la puesta en cuestión de las formas de objetivación. Tal como Foucault lo hiciera de manera recurrente, por ejemplo, respecto de la constitución de la delincuencia, la sexualidad, la anormalidad, el mercado, el homo æeconomicus y el capital humano. Abordaje de los saberes acerca de "lo humano", que, en lugar de cuestionar su "falta de objetividad" y contraponer un "modo adecuado de conocer", se ejercita por medio de la problematización acerca de la formación de dichos objetos, y así desplegar un conjunto de herramientas para la crítica epistemológica de las ciencias humanas que se encuentra jalonada por la preocupación ontológico-política respecto de la constitución de la actualidad. De este modo, en lugar de "la denuncia" del carácter ideológico, sesgado o pseudocientífico de estos saberes, encontramos en la crítica foucaultiana una indagación acerca de la manera en que la formación de estas ciencias contribuye a la constitución de focos de experiencia, articulándose con el ejercicio del poder por medio de prácticas, como el examen y la disciplina, la regulación biopolítica y la gubernamentalidad liberal y neoliberal.

\section{Bibliografía}

Blanchot, Maurice. Michel Foucault tel que je l'imagine. París: Éditions Fata Morgana, 1986.

Brossat, Alain. "Larchive et les archives: Archéologie des discours et gouvernement des vivants". Materiali Foucaultiani 2, n. 4 (2013): 237-254.

Castro, Edgardo. Pensar a Foucault: Interrogantes filosóficos de La arqueología del saber. Buenos Aires: Biblos, 1995.

Castro, Edgardo. Lecturas foucaulteanas: Una historia conceptual de la biopolítica. Buenos Aires: UNIPE Editorial Universitaria, 2011.

Castro-Gómez, Santiago. Historia de la gubernamentalidad: Razón de Estado, liberalismo y neoliberalismo en Michel Foucault. Bogotá: Siglo del Hombre, 2010. 
Defert, Daniel. "Situation du Cours". En Leçons sur la volonté de savoir: Cours au Collège de France, 1970-71, Michel Foucault, 257-279. París: Éditions Gallimard, 2011.

Deleuze, Gilles, Foucault. París: Les Éditions de Minuit, 1986.

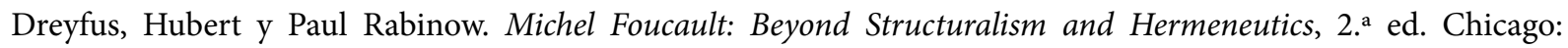
University of Chicago Press, 1983.

Fontana, Alessandro y Mauro Bertani. "Situation du Cours". En "Il faut défendre la société". Cours au Collège de France, 1976, Michel Foucault, 245-263. París: Éditions Gallimard, 1997.

Foucault, Michel. Naissance de la clinique: Une archéologie du regard médical. París: Presses universitaires de France, 1963.

Foucault, Michel. Les mots et les choses: Une archéologie des sciences humaines. París: Éditions Gallimard, 1966.

Foucault, Michel. L’archéologie du savoir. París: Éditions Gallimard, 1969.

Foucault, Michel. Histoire de la folie à l’âge classique. París: Éditions Gallimard, 1972.

Foucault, Michel. Surveiller et punir: Naissance de la prison. París: Éditions Gallimard, 1975.

Foucault, Michel. La volonté de savoir: Histoire de la sexualité 1. París: Éditions Gallimard, 1976.

Foucault, Michel. “Qu’est-ce qu'un philosophe?". En Dits et écrits, 1954-1988. IV. I. 1954-1969, 552-553. París: Éditions Gallimard, 1994.

Foucault, Michel. "La philosophie structuraliste permet de diagnostiquer ce qu'est 'aujourd'hui”. En Dits et écrits, 1954-1988. IV. I. 1954-1969, 580-584. París: Éditions Gallimard, 1994.

Foucault, Michel. "Réponse à une question". En Dits et écrits. 1954-1988. I. 1954-1969, 673-695. París: Éditions Gallimard, 1994.

Foucault, Michel. "Sur l'archéologie des sciences: Réponse au Cercle d'épistémologie". En Dits et écrits. 1954-1988. I. 1954-1969, 696-731. París: Éditions Gallimard, 1994.

Foucault, Michel. "Nietzsche, la généalogie, l'histoire". En Dits et écrits. 1954-1988. II. 1970-1975, 136-156. París: Éditions Gallimard, 1994.

Foucault, Michel. "Les problèmes de la culture, un débat Foucault-Preti". En Dits et écrits. 1954-1988. II. 1970-1975, 369-380. París: Éditions Gallimard, 1994.

Foucault, Michel. "La vérité et les formes juridiques". En Dits et écrits. 1954-1988. II. 1970-1975, 538-646. París: Éditions Gallimard, 1994.

Foucault, Michel. "Il faut défendre la société": Cours au Collège de France, 1976. París: Éditions Gallimard, 1997.

Foucault, Michel. Sécurité, territoire, population: Cours au Collège de France, 1977-78. París: Éditions Gallimard, 2004.

Foucault, Michel. Naissance de la biopolitique: Cours au Collège de France, 1978-79. París: Éditions Gallimard, 2004.

Foucault, Michel. Le gouvernement de soi et des autres: Cours au Collège de France, 1982-1983. París: Éditions Gallimard, 2008.

Foucault, Michel. "Leçon sur Nietzsche". En Leçons sur la volonté de savoir: Cours au Collège de France, 1970-71, 195-213. París: Éditions Gallimard, 2011.

Foucault, Michel. "Résumé du Cours". En Leçons sur la volonté de savoir: Cours au Collège de France, 1970-71, 21522. París: Éditions Gallimard, 2011.

Foucault, Michel. Du gouvernement des vivants: Cours au Collège de France, 1979-80. París: Éditions Gallimard, 2012.

Giordano, Claudia. "Un'idea di filosofia: Michel Foucault, dalla critica dell'antropologia alletica de la cura di sé". Tesis de doctorado. Scuola di Alta Formazione nelle Scienze Umane e Sociali dell'Università degli Studi di Napoli Federico II, 2007.

Gros, Frédéric, "Situation du Cours". En Le gouvernement de soi et des autres: Cours au Collège de France, 1982-1983, Michel Foucault, 347-361. París: Éditions Gallimard, 2008.

Gutting, Gary. Michel Foucault's archaeology of scientific reason. Cambridge: Cambridge University Press, 1989.

Larrauri, Maite. Anarqueología: Foucault y la verdad como campo de batalla. Madrid: Enclave de Libros, 2018.

Le Blanc, Guillaume. La pensée Foucault. París: Ellipses Édition, 2014.

Lehm, Vanessa y Miguel Vatter. "Introduction". En The Government of Life: Foucault, Biopolitics and Neoliberalism, editado por Vanesa Lehm y Miguel Vatter, 1-13. Nueva York: Fordham University Press, 2014.

Mascaretti, Giovanni Maria. "Michel Foucault on Problematization, Parrhesia and Critique". En Materiali Foucaultiani 3, n. ${ }^{\circ}$ 5-6 (2014): 135-154. 
Méndez, Pablo Martín. "Foucault y la arqueología de la política: Tras las huellas de un método inconcluso". Revista de Filosofía Diánoia 65, n. 84 (2020): 81-109.

Paltrinieri, Luca. "Archeologia della volontà: Una preistoria delle Lezioni sulla volontà di sapere”. Quadranti: Rivista Internazionale di Filosofia Contemporanea 2, n. ${ }^{\circ} 1$ (2014): 100-135.

Raffin, Marcelo. "Natureza e política: Uma tensão no pensamento de Michel Foucault". Ágora Filosófica 1, n. 2 (2014): 116-143.

Raffin, Marcelo. "La verdad y las formas políticas: La lectura temprana de la tragedia de Edipo en Michel Foucault". Anacronismo e Irrupción 5 (2015): 54-78.

Sabot, Philippe. Lire Les mots et les choses de Michel Foucault. París: Presses universitaires de France, 2006.

Sacchi, Emiliano. "Umbrales biológicos de la modernidad política en Michel Foucault". Daimon: Revista Internacional de Filosofía, n. ${ }^{\circ} 68$ (2016): 19-35.

Sauquillo González, Julián. "La 'anarqueología' como crítica del gobierno de los hombres por la verdad". En Michel Foucault: Derecho y poder, editado por Mauro Benente, 67-90. Buenos Aires: Didot, 2015.

Wallenstein, Sven-Olov. "Introduction: Foucault, Biopolitics, and Governmentality". En Foucault, Biopolitics and Governmentality, editado por Sven-Olov Wallenstein y Jakob Nilsson, 7-34. Huddinge: Södertörn University, 2013. 